\title{
UV escape fraction and dust distribution of star forming galaxies at $z=0-3$ : a new dust attenuation model
}

\author{
Haruka Kusakabe $^{1}$, Kazuhiro Shimasaku ${ }^{1}$ and Ikkoh Shimizu ${ }^{2}$ \\ ${ }^{1}$ Dept. of Astronomy, Graduate School of Science, The University of Tokyo, 7-3-1 Hongo, \\ Bunkyo-ku, Tokyo 113-0033, Japan; email: kusakabe@astron.s.u-tokyo.ac.jp \\ ${ }^{2}$ Dept. of Earth and Space Science, Graduate School of Science,Osaka University, 1-1 \\ Machikaneyama, Toyonaka-city, Osaka 560-0043, Japan
}

\begin{abstract}
The UV escape fraction, $f_{\mathrm{UV}}^{\mathrm{esc}}$, is a key parameter determining the apparent SED of star forming galaxies. However, it is not well known how $f_{\mathrm{UV}}^{\text {esc }}$ depends on the global geometry of dust distribution, nor how it evolves with time, although several models are proposed (e.g., Calzetti (2001)). We use $\sim 130$ normal star-forming galaxies (114 at $z \sim 0$ from Cortese et al. (2012) and 15 at $z \sim 1-3$ from Magnelli et al. (2012) and Saintonge et al. (2013)), to find that the $z \sim 0$ galaxies show a relatively tight anti-correlation between $f_{\mathrm{UV}}^{\mathrm{esc}}$ and surface dust mass density, $\Sigma_{d}$ (See Fig. 1(a)). This correlation can be reproduced by a dust geometry model that well-mixed stars and dust follow the same exponential profile (Fig. 1(b)) with an effective mass-absorption coefficient $\kappa(1600 \AA)=7.6_{-3.0}^{+5.3} \times 10^{4} \mathrm{~cm}^{2} \mathrm{~g}^{-1}$, similar to the Milky Way value including absorption and scatter. The $z \sim 1-3$ galaxies are not inconsistent with this model. Our model can be easily implemented in semi-analytic models and cosmological hydrodynamics simulations (CHSs) of galaxy formation. Initial results for Shimizu et al. (2014)'s CHSs are presented.
\end{abstract}

Keywords. galaxies: evolution, infrared: galaxies, ultraviolet: galaxies, (ISM:) dust, extinction
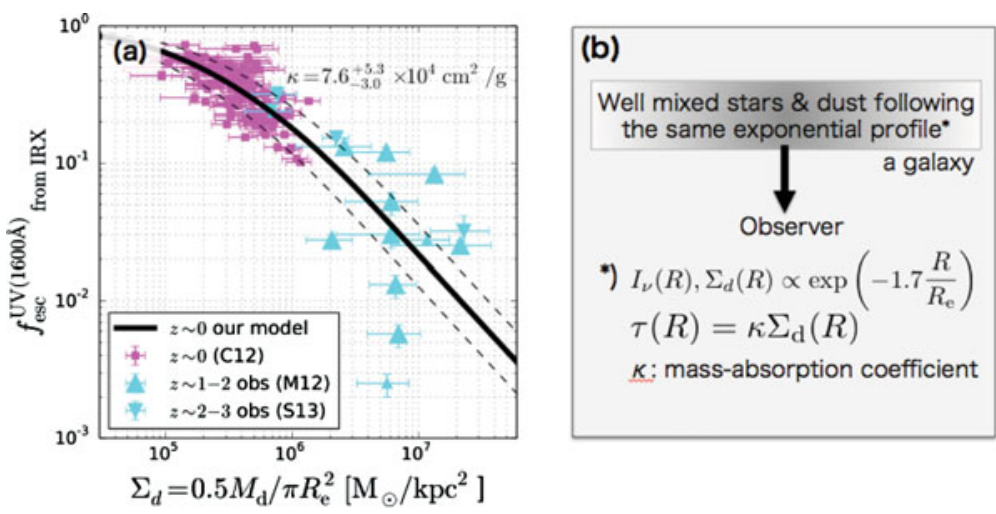

Figure 1. (a) $\Sigma_{\mathrm{d}}$ vs. $f_{\mathrm{UV}}^{\mathrm{esc}}$. The filled circles and triangles represent the data of $z \sim 0-3$ galaxies. The solid line (dashed lines) shows our model with the median (68\%-range) $\kappa$ of the $z \sim 0$ sample. (b) Description of our model.

\section{References}

Calzetti, D. 2001, PASP, 113, 790

Cortese, L., Boissier, S., \& Boselli, A. et al. 2012, A\&GA, 544, 101

Magnelli, B., Saintonge, A., \& Lutz, D. et al. 2012, A\&A A, 548, 22

Saintonge, A., Lutz, D., \& Genzel, R. et al. 2013, ApJ, 778, 2

Shimizu, I., Inoue, K. A., Okamoto, T., \& Yoshida, N. 2014, MNRAS, 440, 731 